\title{
Phonon-emission-accompanied photoluminescence from a two-dimensional electron system at high magnetic field
}

\author{
Vladimir I. Fal'ko* \\ Max-Planck-Institut für Festkörperforschung, Heisenbergstrasse 1, 7000 Stuttgart 80, Germany
}

(Received 4 September 1992)

\begin{abstract}
The magnetic freeze-out of two-dimensional electrons (the formation of a Wigner crystal or disordered dielectric state) is shown to stimulate acoustical- and optical-phonon emissions in the course of their radiative recombination with an acceptor-bound hole. This allows us to expect an appearance of the corresponding phonon replicas in the spectra taken at the long time-delayed tail of the time-resolved magnetoluminescence in low-density $\mathrm{GaAs}-\mathrm{Al}_{x} \mathrm{Ga}_{1-x}$ As heterostructures in extreme magnetic fields.
\end{abstract}

Optical studies of two-dimensional (2D) electrons have been extended in recent years ${ }^{1-3}$ to extremely high quantizing magnetic fields providing a low filling factor where a new localized state of electronic system (treated as crystalline) was found in the transport experiments. ${ }^{4}$ Traditionally, the spectral position of the recombination line of $2 \mathrm{D}$ electrons with a hole is a subject of optical measurements. It provides some fine information on the energetics of electronic system phases, ${ }^{5}$ but tells nothing of localization in this. On the other hand, a dynamical quantity such as the recombination rate can be successfully extracted from the time-resolved magnetoluminescence measurements, ${ }^{6,7}$ and the data reported in ${ }^{8,9}$ show a sudden suppression of recombination of $2 \mathrm{D}$ electrons with a hole bound at an acceptor in the $\delta$-doped layer after the magnetic freeze-out occurs.

This problem can be treated from the point of view of what is known about localized electrons at high magnetic fields ${ }^{10}$ (providing small filling factors $v=2 \pi n_{e} \lambda_{H}^{2}<<1$ ). Indeed, for all electrons being frozen near some centers, the magnetic field squeezes their wave functions to the magnetic length scale $\lambda_{H}$, irrespective of whether the electron localization is caused by a weak impurity ${ }^{10}$ or by crystalline ordering due to the Coulomb interaction. ${ }^{11}$ When the hole is bound at a shallow acceptor (this configuration was studied in Refs. 1, 6, and 8), it forms a neutral complex with it. The correlations between 2D electrons and the hole position can, therefore, be neglected, and recombination of any pair of electrons and hole is suppressed if their separation $R$ is longer than the magnetic length.

Hence, as it was proposed by Shklovskii, ${ }^{10}$ at $R \gg \lambda_{H}$ (which corresponds to the long-time tail) any kind of external perturbation which can break the electron momentum in the course of tunneling reduces the magnetic barrier. In developing Shklovskii's idea, Khaetskii and Matveev have recently suggested ${ }^{12}$ that the phonons in pure systems are possible candidates for such a process. In the present paper, we exploit the possibility of phonon emission during the electron tunneling toward the acceptor-bound hole to predict the appearance of an additional (shifted to the low energies) line at the long delay tail of time-resolved recombination spectra. In what follows, we shall first give a general idea of the phonon emission in the course of magnetotunneling, following the reasoning used in Refs. 10 and 12, and then specify it to the case of optical- and acoustical-phonon emission in high-quality GaAs- $\mathrm{Al}_{x} \mathrm{Ga}_{1-x}$ As heterostructures and estimate the maximal filling factors in the low-density 2D electron gas when this effect can be observed [to anticipate a little, for densities $n_{e} \sim(3-5) \times 10^{10} \mathrm{~s} \mathrm{~m}^{-2}$ these are $v \sim 0.09-0.07$ for acoustical and $v \sim 0.06-0.05$ for optical phonons, respectively].

The quantitative information on relative efficiencies of phonon-accompanied and conventional channels of radiative recombination of a hole from a shallow acceptor with the closest $2 \mathrm{D}$ electron is held by the corresponding rates $\tau_{1}^{-1}$ and $\tau_{0}^{-1}$. We shall consider the radius of a bound hole as the shortest scale in the problem, $\psi_{h}(\mathbf{r})=\sigma^{1 / 2} \delta(\mathbf{r}-\mathbf{R})$, and also assume the electron to be bound, for example, near an infinitely weak center $U=-2 \pi \lambda_{H}^{2} \epsilon_{0} \delta(\mathbf{r})\left(\epsilon_{0}<<\hbar \omega_{c}\right.$ is its binding energy. $\left.{ }^{13}\right)$. A magnetic barrier impedes the direct recombination and the rate $\tau_{0}^{-1}$ is exponentially small, $\tau_{0}^{-1}(R)$ $=\left(\sigma \tau^{-1} / 2 \pi \lambda_{H}^{2}\right) \exp \left(-R^{2} / 2 \lambda_{H}^{2}\right)$ (here $\sigma \tau^{-1}$ is the cross section of a radiative interband transition), so that at the highest fields tunneling with the emission of phonons starts to play a dominant role due to the breakup of a magnetic barrier by the momentum transfer to the lattice. Compared to what takes place in transport, ${ }^{12}$ we can consider the case of a zero temperature, because the energy released in the recombination event exceeds many times over that of the lattice excitations (in GaAs, the gap is about $1.5 \mathrm{eV}$ as compared to the Debay temperature $\sim 40 \mathrm{meV}$ ). For the same reason, the interband matrix element of the optical transition remains the same in spite of a change in irradiated frequency. The calculation below is, therefore, similar to that performed in the studies of magnetotunneling initiated by short-range scatterers, ${ }^{10}$ with the only difference being that the electron loses the energy $\hbar \omega$ ( $\omega$ is the phonon frequency) during the tunneling, which is quite important in the case of optical-phonon emission.

Phonon emission in the course of tunneling. For the sake of simplicity, let us restrict our consideration to only one phonon emitted during the radiative transition. According to the rules of the perturbation theory on 
electron-phonon interaction, a probability of such an event can be written as

$$
\begin{aligned}
\tau_{1}^{-1}(R)=\sigma \tau^{-1} \int & \frac{d q Q_{00}\left(q_{z}\right)|g(q)|^{2}}{(2 \pi)^{3}} \\
& \times\left|\int \frac{e^{-\mathbf{r}^{2} / 4 \lambda_{H}^{2}}}{\sqrt{2 \pi \lambda_{H}^{2}}} G_{\epsilon_{1}}(\mathbf{r}, \mathbf{R}) e^{i \mathbf{q}_{\|} \mathbf{r}} d \mathbf{r}\right|^{2},
\end{aligned}
$$

where $g(q)$ denotes the electron-phonon coupling, and the difference between the retarded and advanced Green functions is obliterated, $G_{\epsilon}=\psi_{n m}^{*}(\mathbf{r})\left[\epsilon-E_{n m}\right]^{-1} \psi_{n m}\left(\mathbf{r}^{\prime}\right)$, by the fact that the electron is always in the classically forbidden region: $\epsilon_{1}=\epsilon_{0}-\hbar \omega<0$ (we pose an energy zero to the Landau level $n=0$, thus $\left.\epsilon_{0} \rightarrow-0\right)$. Further, we use the simplifying assumption that the intersubband splitting $\Delta_{10}$ (the energy difference of confined states in a quantum well forming a $2 \mathrm{D}$ gas) is large enough to avoid virtual transitions to higher subbands from the theory, because they cause sufficient increase of the under-thebarrier action of 2D motion. The factor $Q_{00}\left(q_{z}\right)=\left|\int d z \phi_{0}^{2}(z) e^{i q_{z} z}\right|^{2}$ manifests the resulting separation of in-plane and across-the-plane variables $\left[\phi_{0}(z)\right.$ is the ground subband wave function], and the Green function $G_{\epsilon}$ in Eq. (1) should be treated as a purely 2D one.

According to the symmetry of a problem, we use the axial gauge $\mathbf{A}=-\mathbf{H} \times \mathbf{r} / 2$, with coordinates centered at the initial electron bound-state position, and separate the gauge-dependent phase factor in the Green function, $G_{\epsilon}\left(\mathbf{r}, \mathbf{r}^{\prime}\right)=\exp \left\{i \mathbf{l}_{z}\left(\mathbf{r} \times \mathbf{r}^{\prime}\right) / 2 \lambda_{H}^{2}\right\} F_{\epsilon}\left(\mathbf{r}-\mathbf{r}^{\prime}\right)$, from the radial part $F_{\epsilon}\left(\mathbf{r}-\mathbf{r}^{\prime}\right)$. The latter can be calculated using its definition,
$F_{\epsilon}\left(\mathbf{r}-\mathbf{r}^{\prime}\right)=G_{\epsilon}\left(0, \mathbf{r}^{\prime}-\mathbf{r}\right)$

$$
=\sum_{n, m} \psi_{n m}^{*}(0)\left[\epsilon-E_{n m}\right]^{-1} \psi_{n m}\left(\mathbf{r}^{\prime}-\mathbf{r}\right),
$$

where $\quad \psi_{n m}(0)=0$ for any $m \neq 0, \psi_{n 0}(\mathbf{r})$

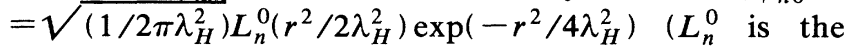
normalized generalized Laguerre polynomial) and $E_{n 0}=n \hbar \omega_{c}$. After some algebra based on the generating function properties of the Laguerre polynomials, $F_{\epsilon}$ takes the form

$$
F_{\epsilon}=\frac{\exp (-\rho)}{2 \pi \lambda_{H}^{2} \hbar \omega_{c}} \int_{0}^{\infty} \frac{d \eta}{\eta} e^{-2 \rho \eta}\left(\frac{\eta}{1+\eta}\right)^{|\epsilon| / \hbar \omega_{c}},
$$

which can be rewritten with the exponential accuracy as $F_{\epsilon} \propto e^{-S}$,

$$
\begin{aligned}
S= & \rho\left[1+\frac{2|\epsilon|}{\hbar \omega_{c} \rho}\right]^{1 / 2} \\
& +\frac{2|\epsilon|}{\hbar \omega_{c}} \ln _{10}\left[\left(\rho+\frac{2|\epsilon|}{\hbar \omega_{c}}\right]^{1 / 2}+\rho^{1 / 2}\right],
\end{aligned}
$$

where $\rho=\left(\mathbf{r}-\mathbf{r}^{\prime}\right)^{2} / 4 \lambda_{H}^{2}$.

In the $N$ order of the perturbation theory of electronphonon interaction, ${ }^{14}$ the amplitude of the process contains $N$ exponential factors like that in Eq. (2). If the magnetic barrier is the main obstacle to the electron tunneling toward a hole, the amplitude is mainly determined by the optimal configuration of electron positions along its path associated with the points where phonons are emitted. ${ }^{12}$ The latter can be found by minimizing the total under-the-barrier action $S_{N}$; for the case of one emitted phonon, $S_{1}$ has the form

$$
S_{1}=\frac{\mathbf{r}^{2}}{4 \lambda_{H}^{2}}+\left\{\frac{(\mathbf{R}-\mathbf{r})^{2}}{4 \lambda_{H}^{2}}\left[1+\frac{2 \omega}{\omega_{c}} \frac{4 \lambda_{H}^{2}}{(\mathbf{R}-\mathbf{r})^{2}}\right]^{1 / 2}+\frac{2 \omega}{\omega_{c}} \ln _{10}\left[\left(\frac{(\mathbf{R}-\mathbf{r})^{2}}{4 \lambda_{H}^{2}}+\frac{2 \omega}{\omega_{c}}\right]^{1 / 2}+\frac{|\mathbf{R}-\mathbf{r}|}{2 \lambda_{H}}\right]\right\}
$$

If one knows the optimal coordinate,

$$
R_{\mathrm{opt}}=\frac{\mathbf{R}}{2}\left[1+\frac{8 \omega}{\omega_{c}} \frac{\lambda_{H}^{2}}{R^{2}}\right],
$$

the action $S_{1}$ can be expanded in the vicinity of $\mathbf{r}=\mathbf{R}_{\mathrm{opt}}$ into the series

$$
\begin{aligned}
S_{1}= & S_{1}\left(\mathbf{R}_{\mathrm{opt}}\right) \\
& +\left[1-\left(8 \omega / \omega_{c}\right)\left(\lambda_{H}^{2} / R^{2}\right)\right]^{-1}\left(\mathbf{r}-\mathbf{R}_{\mathrm{opt}}\right)^{2} / 2 \lambda_{H}^{2}+\cdots
\end{aligned}
$$

When one neglects the phonon frequency, as was done in Ref. $12, \mathbf{R}_{\text {opt }} \approx \mathbf{R} / 2$, whereas the great enough energy loss shifts $\mathbf{R}_{\text {opt }}$ to the end of the tunneling path.

So, the minimal action value $S_{1}\left(\mathbf{R}_{\text {opt }}\right)$ can be picked out of the integral in Eq. (1), $\tau_{1}^{-1} \propto \exp \left\{-2 S_{1}\left(\mathbf{R}_{\text {opt }}\right)\right\}$, and the preexponence can be represented as the Gaussian-like integral over local coordinates $\mathbf{x}=\mathbf{r}-\mathbf{R}_{\mathrm{opt}}$ and $\mathbf{x}^{\prime}=\mathbf{r}^{\prime}-\mathbf{R}_{\text {opt }}$. Performing the $\mathbf{x}$ and $\mathbf{x}^{\prime}$ integrals, we find the preexponence in the form

$$
\begin{aligned}
& \int \frac{d \mathbf{q}_{\|} d q_{z}|g(q)|^{2} Q_{00}\left(q_{z}\right) \lambda_{H}^{2}}{2 \pi(\hbar \omega)^{2}}\left[1-\frac{8 \omega}{\omega_{c}} \frac{\lambda_{H}^{2}}{R^{2}}\right]^{2} \\
& \quad \times \exp \left\{-\left[1-\frac{8 \omega}{\omega_{c}} \frac{\lambda_{H}^{2}}{R^{2}}\right]\left[\mathbf{q}_{\|} \lambda_{H}-\frac{\mathbf{R} \times \mathbf{1}_{z}}{2 \lambda_{H}}\right]^{2}\right\},
\end{aligned}
$$

where the appearance of the last terms owes to the gauge multiplier in $G_{\epsilon}\left(\mathbf{r}, \mathbf{r}^{\prime}\right)$.

Before integrating over the phonon variables $q$, we note that the exponential multiplier in this integral extracts the values $\mathbf{q}_{\|} \approx \mathbf{R} \times \mathbf{1}_{z} / 2 \lambda_{H}^{2}$ with the accuracy of $\delta q \sim \lambda_{H}^{-1} / \sqrt{1-\left(8 \omega / \omega_{c}\right)\left(\lambda_{H}^{2} / R^{2}\right)}$ and can be replaced formally by the corresponding $\delta$ function, if $\delta q \ll</ 2 \lambda_{H}^{2}$. When $\omega$ is negligibly small, $\delta q \sim \lambda_{H}^{-1}$ and the latter condition is obviously satisfied. When $\omega \gg \omega_{c}$, which takes place in optical-phonon emission, we should 
take into account that the enhanced phonon emission can be expected only if the action $S_{1}$,

$S_{1}\left(\mathbf{R}_{\mathrm{opt}}\right)=\frac{R^{2}}{8 \lambda_{H}^{2}}+\frac{\omega}{\omega_{c}}\left[1+2 \ln _{10}\left(\frac{R}{2 \lambda_{H}}+\frac{4 \omega}{\omega_{c}} \frac{\lambda_{H}}{R}\right)\right]$,

is smaller than the under-the-barrier action $S_{0}=R^{2} / 4 \lambda_{H}^{2}$ taken during the direct tunneling. Under such a condition,

$(\delta q / q)<\left[\left(4 \omega / \omega_{c}\right) \ln _{10}\left(R / 2 \lambda_{H}+4 \omega \lambda_{H} / R \omega_{c}\right)\right]^{-1 / 2}<<1$.

Furthermore, the momentum $q$ is usually greater than the inverse width $\lambda_{z}^{-1}$ of a well; thus we substitute into the above equations the effective coupling constant $g\left(R / 2 \lambda_{H}^{2}\right)$ instead of the interaction amplitude $g(q)$, and finally find the volume of the phonon phase space used in the process as $\int Q_{00}\left(q_{z}\right) d q_{z} / 2 \pi=\int d z \phi_{0}^{4}(z) \sim \gamma_{0} / \lambda_{z}$. [For instance, in a triangular well, $\lambda_{z}$ can be related to the intersubband splitting $\Delta_{10}$ as $\lambda_{z}^{-1} \approx\left(2 m \Delta_{10} /\right.$ $\left.1.75 \hbar^{2}\right)^{1 / 2}$, and the numerical factor is $\gamma_{0} \sim 0.4$. ]

Emission of a longitudinal-optical (LO) phonon. The energy released to the lattice due to the LO-phonon emission depends slightly on the phonon momentum (for example, in GaAs the optical-phonon energy is $\left.\hbar \omega=\hbar \omega_{o} \approx 36 \mathrm{meV}\right)$. The latter is already determined as $q \approx R / 2 \lambda_{H}^{2}$, so that the corresponding radiative recombination rate has the form

$$
\tau_{1}^{-1}(R)=\frac{1}{2} \frac{\sigma \tau^{-1}}{2 \pi \lambda_{H}^{2}} \gamma_{0} \alpha_{o} e^{-2 S_{1}\left(\mathbf{R}_{\mathrm{opt}}\right)}
$$

where the dimensionless parameter

$$
\alpha_{o}=\frac{(e \Gamma)^{2} / \lambda_{z}}{2 \pi \hbar \mu \omega_{o}^{3}} \frac{2 \lambda_{H}^{2}}{R^{2}}
$$

describes the efficiency of the $2 \mathrm{D}$ electron polar coupling with the LO lattice mode ( $e \Gamma$ is the coupling constant, $\mu$ is the unit-cell mass). In GaAs, in the important range of $q$, the parameter $\alpha_{o}$ is small. The emission of phonons is stimulated, therefore, only when the ratio $R / \lambda_{H}$ is great enough, so in our analysis we refer to the structures with the lowest electron density $n_{e}=(3-5) \times 10^{10} \mathrm{~s} \mathrm{~m}^{-2}$ used in optical experiments. ${ }^{6,7}$ In this case $\lambda_{z} \sim 100 A$, and using the material constants taken from the tables in Ref. 15 we estimate $\alpha_{o} \sim 0.1\left(\lambda_{H} / R\right)^{2}$.

The separation $R$ between the electron and hole is limited by the value $\approx 1 / \sqrt{\pi n_{e}}$. Substituting this value into Eq. (5), we find a maximal filling factor $v \approx 2 \lambda_{H}^{2} / R^{2}$, which allows the rate $\tau_{1}^{-1}$ to be greater than the direct recombination rate $\tau_{0}^{-1}$. This estimation gives the "critical" filling factor, when one phonon can be emitted from the range of $v \sim 0.06-0.05$. Emission of more than one LO phonon is suppressed for the reason mentioned above. Hence, at filling factors less than 0.05 in the lowdensity GaAs- $\mathrm{Al}_{x} \mathrm{Ga}_{1-x}$ As heterostructures we should expect a single LO-phonon replica at the long delay tail in the time-resolved magnetoluminescence spectra.

Emission of an acoustical phonon. Recombination accompanied by emission of an acoustical phonon comprises a competing channel to the recombination accompanied by the optical phonons. For the sake of simplicity, we do not distinguish sound velocities $s$ of longitudinal- and transverse-acoustical modes, $\omega=s q$; we also consider the electron-phonon coupling as produced both by polarization and deformation potentials of a wave in the cubic polar crystal (like GaAs). The corresponding radiative rate has a form completely analogous to that in Eq. (5), with the only difference being that the action determined by Eq. (4) can be simplified to $S_{1}\left(R_{\text {opt }}\right)=R^{2} / 8 \lambda_{H}^{2}+m s R \hbar^{-1} \ln _{10}\left(R / \sqrt{8} \lambda_{H}\right)$, and parameter $\alpha_{o}$ should be replaced by the one describing acoustical phonons:

$$
\alpha_{a}=\frac{\Xi^{2} /\left(s R \lambda_{z}\right)}{4 \pi \hbar \mu s^{2}}\left[1+\left(\frac{e \beta \lambda_{H}^{2}}{\Xi(R / 2)}\right)^{2}\right]
$$

( $\Xi$ and $e \beta$ are deformation and polarization interaction constants). In GaAs, the deformation mechanism of coupling is dominant for the important range of phonon momenta. Turning to the low-density structures $n_{e}=(3-5) \times 10^{10} \mathrm{~s} \mathrm{~m}^{-2}$ used in optical experiments, ${ }^{6,7}$ we estimate $\alpha_{a} \sim 10^{-3}$ and find that the loss of energy is too weak to give the significant value of the second term in the exponence in the rate $\tau_{1}^{-1}\left(m s R \hbar^{-1}<0.15\right)$.

Reproducing the same estimations as above, we find that the emission of an acoustical phonon starts first at the filling factor $v \sim 0.09-0.07$. In optics, this phonon emission should manifest itself as an additional line shifted to the low energies into the value

$$
\Delta_{1}=\hbar s \frac{R}{2 \lambda_{H}^{2}} \sim \frac{\hbar s}{\lambda_{H} \sqrt{2 v}} \sim(2-5) \mathrm{meV} .
$$

This shift is beyond the linewidth in the high-quality samples. ${ }^{1-3}$ It is, therefore, observable in spectra taken at the long delay tail of the time-resolved magnetoluminescence.

For lower fillings, more than one acoustical phonon can be emitted in the course of recombination. The rate of this process can be calculated (with the preexponential accuracy) using the optimal trajectory method, ${ }^{12}$ and (for a given $v$ ) the maximal number of emitted acoustical phonons can be found as $N \approx\left[v\left|\ln _{10}\left(\gamma_{0} \alpha_{a}\right)\right|\right]^{-1}$. As to the position of the phonon repetition line in recombination spectra, the increase of a number of emitted phonons does not change its shift $\Delta_{N}$ strongly, because the electron displacement limits the summed energy transferred to the lattice,

$$
\Delta_{N}=\sum_{j=1}^{N} \hbar \omega_{j} \approx \hbar s N R /(N+1) \lambda_{H}^{2} \leq \hbar s / \lambda_{H} \sqrt{v / 2} .
$$

Reverting to the photoluminescence studies in the Wigner solid phase of $2 \mathrm{D}$ electrons, ${ }^{8}$ we claim that the above analysis also remains valid in this regime. At high fields, electrons occupy the lowest Landau level, and without any interaction their ground state is infinitely degenerate. The Coulomb interaction breaks this degeneracy, and, even if it is quite weak in comparison with the cyclotron energy, the electrostatic potential $U(\mathbf{r})$ created by all the other electrons from the Wigner lattice localizes recombining a particle (already squeezed by the magnetic field) in the middle of its cell. We can, therefore, re- 
peat all the above arguments in accounting for a smooth 2D potential $U(r)$ in addition to the magnetic barrier. In a harmonic approximation ${ }^{16}$ (which is reasonable for our aims ${ }^{17}$ ), this potential has the form

$$
U(r) \approx \frac{e^{2}}{\chi} \sum_{k \neq 0} \frac{3\left(\mathbf{y}_{k} \mathbf{r}\right)^{2}-\mathbf{y}_{k}^{2} r^{2}}{2\left|\mathbf{y}_{k}\right|^{5}} \approx \frac{3 \pi^{3 / 2}}{16} \frac{e^{2} n_{e}^{1 / 2}}{\chi} r^{2} n_{e},
$$

where $\left\{\mathbf{y}_{k}\right\}$ are sites of the triangular Wigner lattice and $\chi$ is dielectric constant of a semiconductor. In estimating the efficiency of an additional barrier we substitute $r \approx\left(\pi n_{e}\right)^{-1 / 2}$ and find $U$ to be about a few $\mathrm{meV}$ for $n_{e} \sim 10^{10-11} \mathrm{~s} \mathrm{~m}^{-2}$, while $\hbar \omega_{c} \sim 10 \mathrm{meV}$ in the region of fields where the crystal can be stable; ${ }^{11,18-20}$ a change in the effective tunneling barrier curvature is also slight. That means that the acoustical-phonon emission in the crystalline state has the same threshold as that estimated for localization caused by weak centers.

In summary, we predict the appearance (at filling factors $v \sim 0.09-0.07$ ) of an acoustical phonon and (at lower filling $v \sim 0.06-0.05$ ) longitudinal optical-phonon replicas at the long delay tail of the time-resolved magnetoluminescence spectra in the low-density GaAs$\mathrm{Al}_{x} \mathrm{Ga}_{1-x} \mathrm{As}$ heterostructures in quantizing magnetic fields, which should manifest the short-range localization of particles in a magnetically frozen state (for example, in the Wigner crystalline state).

The author thanks Dr. A. Khaetskii, Dr. I. Kukushkin, and Dr. N. Pulsford for stimulating discussions. The author also acknowledges support from the Alexander von Humboldt Foundation.
*On leave from Theory Department, Institute of Solid State Physics, Russian Academy of Sciences, Cherusgolovka 142432, Russia.

${ }^{1}$ H. Buhmann, W. Joss, K. v. Klitzing, I. V. Kukushkin, G. Martinez, A. Plaut, K. Ploog, and V. B. Timofeev, Phys. Rev. Lett. 66, 926 (1991).

${ }^{2}$ B. B. Goldberg, D. Heiman, A. Pinczuk, L. Pfeiffer, and K. W. West, Surf. Sci. 263, 9 (1992).

${ }^{3}$ A. J. Turberfield, S. R. Haynes, P. A. Wright, R. A. Ford, R. G. Clark, J. F. Ryan, J. J. Harris, and C. T. Foxon, Surf. Sci. 263, 1 (1992); E. M. Goldys, S. A. Brown, R. B. Dunford et al., Phys. Rev. B 46, 7957 (1992).

${ }^{4}$ E. Y. Andrei et al., Phys. Rev. Lett. 60, 2765 (1988); R. L. Willet et al., Phys. Rev. B 38, 7881 (1988); H. W. Jiang et al., Phys. Rev. Lett. 65, 633 (1990); V. J. Goldman et al., ibid. 65, 2189 (1990); M. A. Paalanen et al., Phys. Rev. B 45, 13784 (1992).

${ }^{5}$ V. M. Apal'kov and E. I. Rashba, Pis'ma Zh. Eksp. Teor. Fiz. 53, 420 (1991) [JETP Lett. 53, 442 (1991)]; A. H. MacDonald, E. H. Rezayi, and D. Keller, Phys. Rev. Lett. 68, 1939 (1992); B. S. Wang, J. L. Birman, and Z. B. Su, ibid., 68, 1605 (1992).

${ }^{6}$ A. F. Dite, I. V. Kukushkin, V. B. Timofeev, A. I. Filin, and K. v. Klitzing, Pis'ma Zh. Eksp. Teor. Fiz. 54, 635 (1991) [JETP Lett. 54, 642 (1991)].

${ }^{7}$ M. Dahl, D. Heiman, A. Pinczuk, B. B. Goldberg, L. N. Pfeiffer, and K. W. West, Phys. Rev. B 45, 6957 (1992).

${ }^{8}$ I. V. Kukushkin et al., Europhys. Lett. 18, 63 (1992).

${ }^{9}$ I. V. Kukushkin, N. J. Pulsford, K. v. Klitzing, R. Haug, K. Ploog, and V. B. Timofeev (unpublished).

${ }^{10}$ B. I. Shklovskii, Pis'ma Zh. Eksp. Teor. Fiz. 36, 43 (1982) [JETP Lett. 36, 51 (1982)]; B. I. Shklovskii and A. L. Efros,
Zh. Eksp. Teor. Fiz. 84, 811 (1983) [Sov. Phys. JETP 57, 470 (1983)].

${ }^{11}$ K. Maki and X. Zotos, Phys. Rev. B 28, 4349 (1983).

${ }^{12}$ A. V. Khaetskii and K. A. Matveev, Phys. Rev. B 44, 3444 (1991); A. V. Khaetskii and K. A. Matveev, J. Phys. Condens. Matter 4, 3491 (1992).

${ }^{13}$ L. D. Landau and E. M. Litshitz, Quantum Mechanics (Pergamon, Oxford, 1977), pp. 458-460.

${ }^{14}$ T. Holstein, Phys. Rev. 113, 479 (1959).

${ }^{15}$ V. F. Gantmakher and I. B. Levinson, in Carrier Scattering in Metals and Semiconductors, Modern Problems in Condensed Matter Science, edited by V. M. Agranovich and A. A. Maradudin (North-Holland, Amsterdam, 1987).

${ }^{16}$ H. Fukuyama, Solid State Commun. 17, 1323 (1975).

${ }^{17}$ The self-consistent calculation of the crystalline wave functions (Ref. 18) has shown a weak renormalization of the electron localization length near the site as compared to $\lambda_{H}$ in the regime when the Coulomb energy is much smaller than the cyclotron one. The exchange corrections found in Ref. 18 are negligible and the wave functions are very similar to Maki and Zotos's one (Ref. 11), which just corresponds to what is obtainable in the harmonic approximation (Ref. 19).

${ }^{18}$ P. K. Lam and S. M. Girvin, Phys. Rev. B 30, 473 (1984); D. Leveque, J. J. Weis, and A. H. MacDonald, ibid. 30, 1056 (1984).

${ }^{19}$ F. P. Ulinich and N. A. Usov, Zh. Eksp. Teor. Fiz. 76, 228 (1978) [Sov. Phys. JETP 49, 147 (1978)].

${ }^{20}$ R. B. Laughlin, Phys. Rev. Lett. 50, 1395 (1983); D. Yoshioka and P. A. Lee, Phys. Rev. B 27, 4983 (1983); K. Esfarjani and S. T. Chui, ibid. 42, 10758 (1990). 